\title{
Assessment of the Characteristics and Detectability of Skin Perfusion Pressure Measured Using a Thermostatic Heating Probe
}

\author{
Yoshiko Watanabe, MD, PhD, ${ }^{1}$ Hisao Masaki, MD, PhD, ${ }^{2}$ Kenji Kojima, ${ }^{3}$ Atushi Tabuchi, MD, PhD, ${ }^{2}$ \\ Yasuhiro Yunoki, MD, PhD, ${ }^{2}$ Hiroshi Furukawa, MD, PhD, ${ }^{2}$ Takahiko Yamasawa, MD, PhD, ${ }^{2}$ \\ Hiroki Takiuchi, MD, ${ }^{2}$ Takeshi Honda, MD, ${ }^{2}$ Noriaki Kuwada, MD, ${ }^{2}$ and Kazuo Tanemoto, MD, $\mathrm{PhD}^{2}$
}

Objectives: To assess the characteristics of skin perfusion pressure (SPP) measured using a thermostatic heating probe and whether a thermostatic heating probe improves SPP detection.

Methods: We studied 8 feet of healthy young subjects and 31 feet of elderly patients suspected to have severe limb ischemia. We measured SPP at the dorsum and plantar aspects of each foot using a plain laser Doppler probe and a thermostatic heating probe heated at $44^{\circ} \mathrm{C}$. Results were expressed as median. Comparisons were analyzed using a non-parametric test.

Results: In the healthy subjects, the SPP values at both the dorsum and the plantar aspect were not significantly different after heating. The thermostatic heating probe did not improve the SPP detection rates. In the patients with ischemic limb, the SPP values at both the dorsum and the plantar aspect significantly increased after heating $(\mathrm{p}<\mathbf{0 . 0 0 1}$ for both). The SPP detection rate at the dorsum remained at $\mathbf{9 6 . 8 \%}$; however, it was improved from $87.1 \%$ to $100 \%$ at the plantar aspect after heating.

Conclusion: The thermostatic heating probe was shown to be useful for improving the detectability of SPP in the ischemic limbs. An SPP increase after heating may be considered as a parameter of limb ischemia.

Keywords: skin perfusion pressure, skin temperature, thermostatic heating probe, response by heating, critical limb ischemia

\section{INTRODUCTION}

$\mathrm{S}_{\mathrm{k}}^{\mathrm{k}}$ kin perfusion pressure (SPP) measurement using a laser Doppler perfusion monitor is useful for evaluating limb ischemia. However, SPP is usually difficult

\footnotetext{
${ }^{1}$ Department of Physiology 1, Kawasaki Medical School, Kurashiki, Okayama, Japan

${ }^{2}$ Division of Cardiovascular Surgery, Department of Surgery, Kawasaki Medical School, Kurashiki, Okayama, Japan

${ }^{3}$ Vascular Laboratory of Kawasaki Medical School Hospital,

Kurashiki, Okayama, Japan

Received: July 2, 2013; Accepted: October 10, 2013

Corresponding author: Yoshiko Watanabe, MD, PhD. Department of

Physiology 1, Kawasaki Medical School, 577 Matsushima,

Kurashiki, Okayama 709-0192, Japan

Tel: +81-86-462-1111, Fax: +81-86-464-1189

E-mail: yosiyosi515@mac.com
}

to detect in limbs with severe ischemia. Skin perfusion is strongly dependent on temperature and environment. Among patients with limb ischemia, some have very poor skin perfusion as SPP can hardly be detected. On the other hand, there are also many patients whose low skin temperature makes SPP detection in their limbs even more difficult.

To measure SPP in an ideal condition, Castronuovo, et al. used a warm heating pad for 15 minutes to elevate the baseline skin perfusion to reach the detectable level by a laser Doppler monitor before the measurement. ${ }^{1)}$ For SPP measurement, the examination room must be warm and the limbs must be properly warmed. However, even in an adequately warmed examination room, it remains troublesome and time-consuming to warm cold limbs, particularly for outpatients.

Skin perfusion is associated with surface temperature 
Table 1 Demographic characteristics and backgrounds of the subjects

\begin{tabular}{lccc}
\hline Group & Healthy subjects & Patients & p-value \\
\hline Number of feet & 8 & 31 & \\
Male:Female & $4: 4$ & $16: 15$ & $>0.99$ \\
Age, median years (range) & $21(20-21)$ & $85(57-93)$ & $<0.001^{* *}$ \\
Ankle pressure (mmHg)* & & $54(20-82)$ & \\
ABI* & & $0.34(0.05-0.68)$ & \\
Toe pressure (mmHg)* & $0(0-44)$ & \\
TBI* & & $0(0-0.35)$ & \\
Rest pain, $\mathrm{n}(\%)$ & $18(58.1)$ & \\
Ulcer, $\mathrm{n}(\%)$ & $9(29.0)$ & $0.001^{* *}$ \\
Background disease, $\mathrm{n}(\%)$ & & $22(71.0)$ & 0.16 \\
Hypertension & 0 & $11(35.5)$ & 0.56 \\
Hyperlipidemia & 0 & $9(29.0)$ & \\
Diabetes & 0 & $4(12.9)$ & \\
Renal disorder $(\mathrm{Cr}>2.0 \mathrm{mg} / \mathrm{dl})$ & 0 & $0(0)$ & \\
-receiving hemodialysis & 0 & & \\
\hline
\end{tabular}

*: median (interquartile range), **: Significant difference. ABI: ankle brachial pressure index;

TBI: toe brachial pressure index

and core body temperature, and heating the local skin can increase skin perfusion..$^{2-5}$ Therefore, a thermostatic heating probe, which can quickly heat an attached skin area at $44^{\circ} \mathrm{C}$, may help in SPP measurement. ${ }^{4,6)}$

In this study, we assessed the characteristics of SPP measurement using a thermostatic heating probe in the feet of healthy young subjects and patients with ischemic limbs, and evaluated whether a thermostatic heating probe can improve SPP detectability.

\section{Materials And Methods}

Between February and May 2012, we measured SPP in 8 feet of eight healthy volunteers and 31 feet of 18 patients suspected to have severe limb ischemia using a thermostatic heating probe. The patients were recruited when 1 or more of the following conditions were observed on the foot: chronic ischemic rest pain or ulcer, ankle pressure lower than $50 \mathrm{mmHg}$, or toe pressure lower than $30 \mathrm{mmHg}$. The patient data collected included age, gender, ankle pressure, toe pressure, and comorbidities (i.e., hypertension, hyperlipidemia, chronic kidney disease, and diabetes).

SPP was measured in our vascular laboratory where the temperature was maintained at $25^{\circ} \mathrm{C}$ with the subjects in the supine position using PeriFlux system 5000 (Perimed AB, Sweden). In each foot, we used 2 types of laser Doppler probe, a standard plain laser Doppler probe and a thermostatic heating probe that heats up to $44^{\circ} \mathrm{C}$, positioned underneath a $5.8-\mathrm{cm}$-wide blood pressure cuff wrapped around the foot. We initially measured
SPP using a plain probe at the dorsum and plantar aspects and then measured similarly using a thermostatic heating probe. When using the thermostatic heating probe, measurement was started after waiting for about 10-20 seconds until the baseline blood flow volume was stabilized. The pressure cuff was inflated to $100 \mathrm{mmHg}$ manually and deflated automatically $(3.4 \mathrm{mmHg} / \mathrm{sec})$.

All statistical analyses were performed using JMP statistical software (version 9.0.2; SAS Institute, Cary, North Carolina, USA). Results were expressed as median (interquartile range). Comparison of age between the 2 groups was analyzed using the Wilcoxon rank-sum test, and other characteristics were compared using the Fisher exact test. The differences in SPP between the 2 types of probes and the differences in SPP between the 2 aspects of the foot were analyzed using the Wilcoxon signed-rank test. The effects of background diseases on SPP changes induced by heating of the feet of patients were analyzed using the Wilcoxon rank-sum test. A p-value of less than 0.05 was considered to indicate a statistically significant difference. When the SPP value was undetectable, it was treated as a missing value.

\section{Results}

The characteristics of the patients are summarized in Table 1. The patients were older than the healthy subjects and had a high proportion of hypertension.

We compared the values and detection rates of SPP, which changed according to the probes used (Figs. 1 and 2). In the healthy subjects, the SPP values at the dorsum and 
(A)

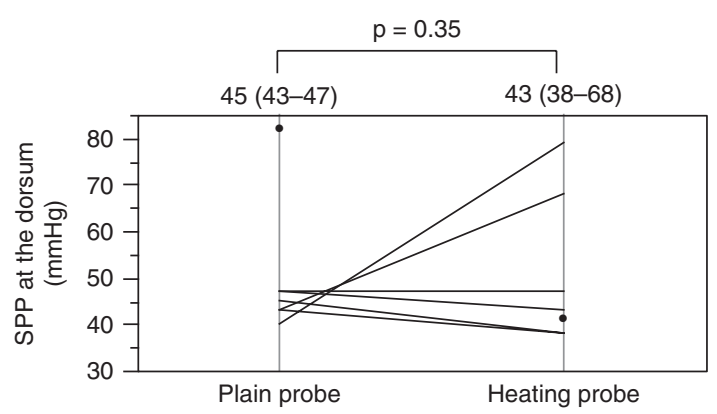

Foot with undetectable SPP; Detectable rate $\mathrm{n}(\%)$

$1(87.5 \%)$

$1(87.5 \%)$

(B)

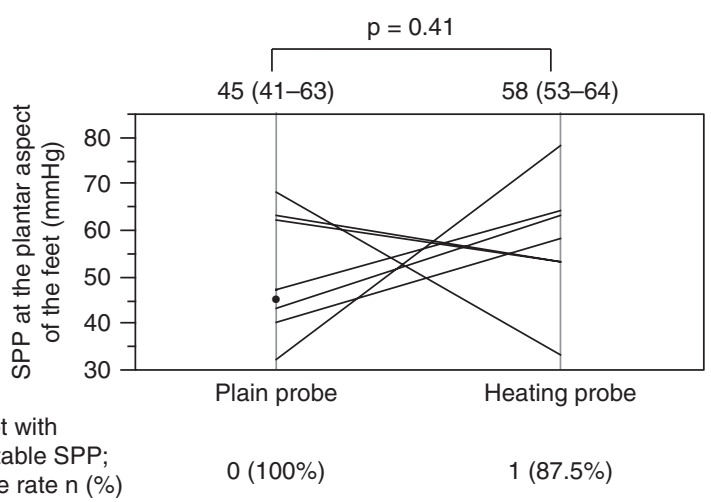

Detectable rate $\mathrm{n}(\%)$

Fig. 1 Skin perfusion pressure (SPP) changes according to the probes used in the feet of the healthy subjects. (A) Differences in SPP values according to the probe used at the dorsum. (B) Differences in SPP values according to the probe used at the plantar aspect. SPP values are expressed as median (interquartile range).

plantar aspects showed no significant difference between the plain laser Doppler probe and the thermostatic heating probe $(\mathrm{p}=0.35$ and $\mathrm{p}=0.41$, respectively). At the dorsum, the SPP detection rates were equal (87.5\%) because there was only 1 foot with undetectable SPP for each probe (Fig. 1A). The SPP detection rate at the plantar aspect was $100 \%$ when using the plain laser Doppler probe, but it decreased to $87.5 \%$ when using the thermostatic heating probe (Fig. 1B).

In the feet of the patients, both SPP values at the dorsum and plantar aspects of the feet increased significantly when the thermostatic heating probe was used (Fig. 2A and $2 \mathrm{~B} ; \mathrm{p}<0.001$ for both). At the dorsum, there was 1 foot in which SPP was undetectable even when the thermostatic heating probe was used; the SPP detection rate was $96.8 \%$ using both probes (Fig. 2A). The SPP detection rate was $87.1 \%$ when the plain laser Doppler probe was used (number of feet with undetectable SPP $=4$ ), but the rate reached $100 \%$ when a thermostatic heating probe was used (Fig. 2B).
(A)

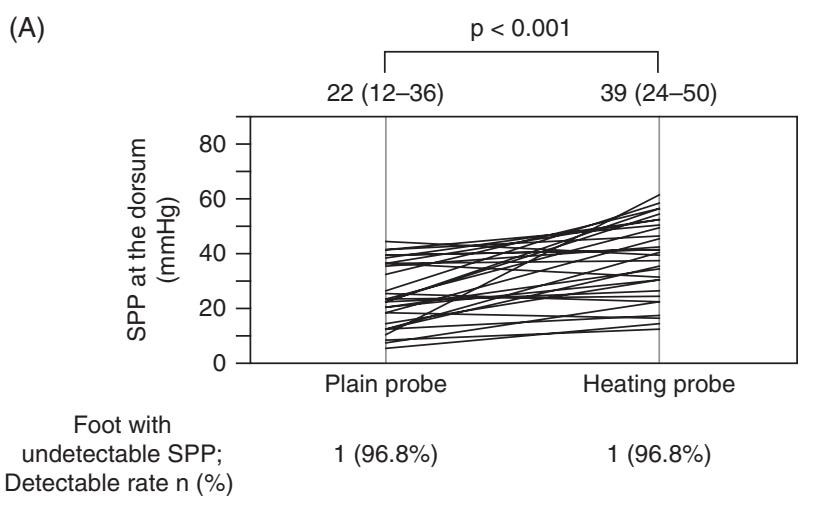

(B)

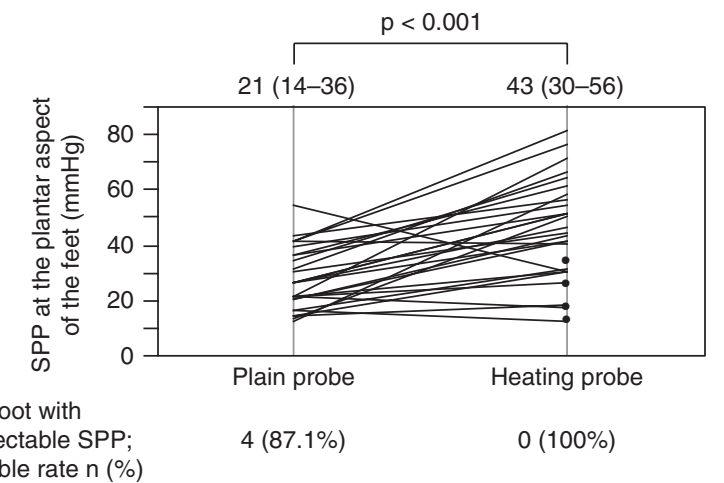

Detectable rate $\mathrm{n}(\%)$

Fig. 2 Skin perfusion pressure (SPP) changes according to the probes used in the feet of the patients with ischemic limbs. (A) Differences in SPP values according to the probe used at the dorsum. (B) Differences in SPP values according to the probe used at the plantar aspect.

There were 7 feet that showed an SPP of $30 \mathrm{mmHg}$ or less at both aspects whichever probe was used, including 5 with ulcer. The 2 feet with ulcer belonged to 1 patient who had diabetes and renal disorder and who later died in the hospital. In her right foot, the SPP was undetectable at the dorsum whichever probe was used. There were 4 feet in which SPP became detectable using the thermostatic heating probe. Of these, 3 feet were the remaining feet with ulcer that showed an SPP of $30 \mathrm{mmHg}$ or less in both aspects whichever probe was used; 1 of these needed major amputation. The remaining 1 foot, in which SPP became detectable as $34 \mathrm{mmHg}$ after heating, did not have ulcer. There were 6 feet that showed an SPP of $50 \mathrm{mmHg}$ or more at both aspects when the thermostatic heating probe was used; these feet did not have ulcer. Although three of them were observed, no patient developed ulcer during their hospitalization. There were no statistically significant effects of comorbidities on SPP increase.

Figure 3 shows the comparisons of the SPP values between the aspects of the feet of the healthy subjects 
(A)

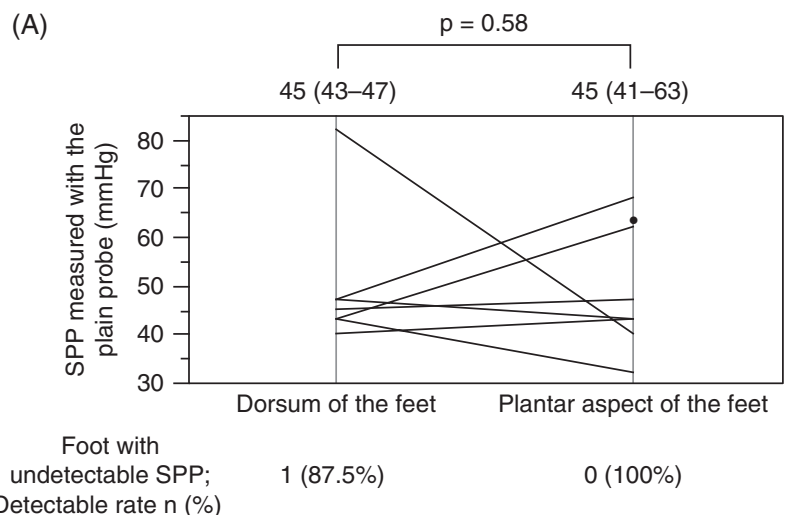

(B)

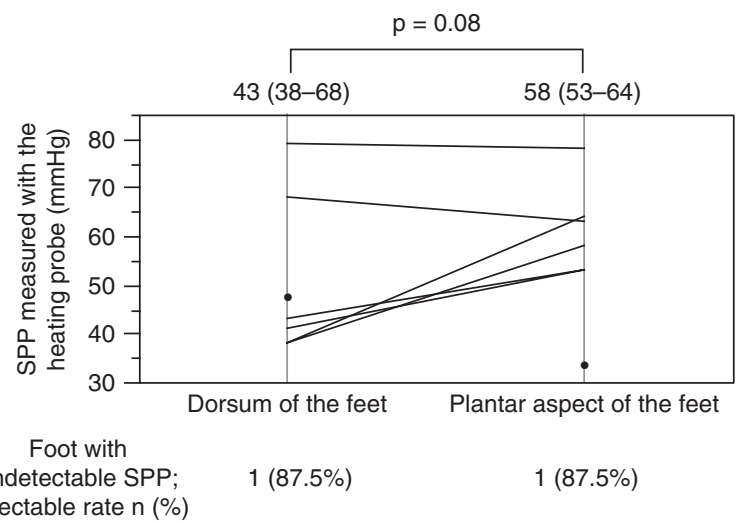

Fig. 3 Comparison of the skin perfusion pressure (SPP) values between the aspects of the feet of the healthy subjects. (A) SPP values measured using the plain laser Doppler probe. (B) SPP values measured using the thermostatic heating probe.

measured using the plain laser Doppler probe and thermostatic heating probe. Figure 4 shows the same comparisons of the patients. Regardless of the probes used, the SPP values were not significantly different between the dorsum and plantar aspects in each group.

\section{Discussion}

SPP measurement is affected by skin temperature. SPP is difficult to measure in cold ischemic limbs and requires limbs to be warmed before measurement. ${ }^{1)}$ Castronuovo, et al. used a warm heating pad for 15 minutes when baseline skin perfusion was less than 0.3 volume $\%$ units before the measurement. ${ }^{1)}$ In contrast, the thermostatic heating probe can elevate and stabilize skin perfusion in about 1 minute, which is very helpful and advantageous in SPP measurement. Although there are currently no reports regarding SPP measurement using a thermostatic heating probe, we projected that the use of such probe would be convenient to improve SPP measurement in ischemic limbs.
(A)

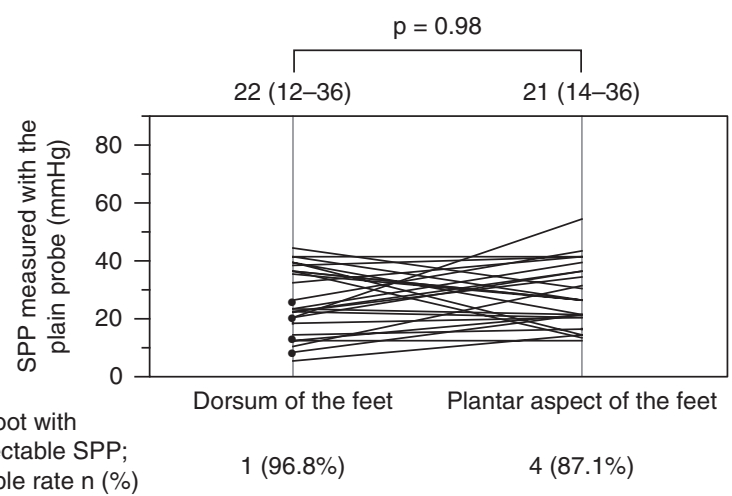

(B)

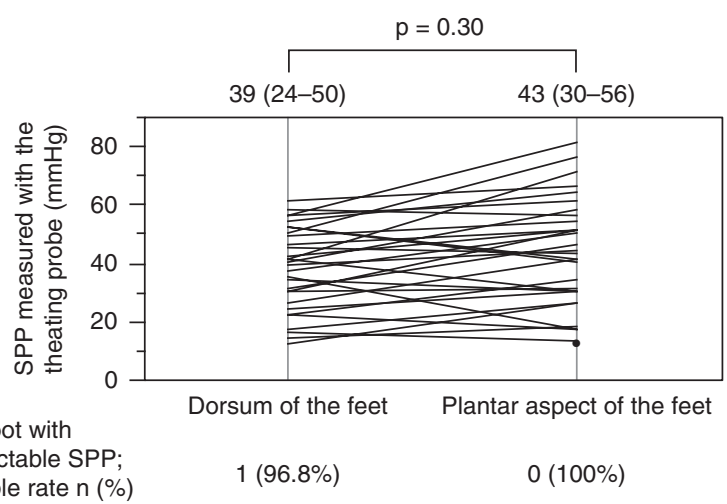

Fig. 4 Comparison of the skin perfusion pressure (SPP) values between the aspects of the feet of the patients with ischemic limbs. (A) SPP values measured using the plain laser Doppler probe. (B) SPP values measured using the thermostatic heating probe.

We investigated the characteristics and detection rates of SPP measurement using a plain laser Doppler probe and a thermostatic heating probe in young healthy subjects and patients suspected to have severe limb ischemia. In the healthy subjects, the SPP values and the changes after heating varied, with 3 subjects showing an undetectable SPP in any 1 of 4 measurements. On the other hand, the detectability of SPP was improved at the plantar aspect of the feet in the patients when the thermostatic heating probe was used. The median SPP values increased in the feet of the patients after heating.

Skin perfusion is controlled by a sympathetic mechanism and a non-sympathetic local mechanism. Previous studies have shown that skin perfusion is increased by artificial heating, with a large increase in young people, but is decreased in elderly people. ${ }^{7,8}$ Nitric oxide (NO) production of the vascular endothelium is considered to be one of the non-sympathetic mechanisms of capillary dilation induced by heating;,59) therefore, the response to heating is decreased in elderly people. On the other hand, Kingma, et al. reported that aging does not alter 
noradrenergic sensitivity during local cooling at the dorsal and ventral sides of the hand, and vasoconstriction by a local mechanism was able to compensate in the hairless skin area when a noradrenergic mechanism is blocked even in elderly subjects. ${ }^{10)}$ As the patients in this study were elderly, sympathetic function might be reduced, and they may also have ischemic damage resulting in auto-sympathetic denervation in their feet, whereas they might maintain local vasoconstriction function in response to coldness. The thermostatic heating probe is considered to induce a non-adrenergic local vasodilation response. Even the ischemic feet of aged patients show redness and warmth when infected. In addition, severe ischemic feet had also shown an increase in skin perfusion in warm water foot bath. ${ }^{11)}$ Also in this study, some ischemic feet in the elderly patients appeared to maintain vasodilation responsiveness to temperature. There were 4 feet in which SPP became detectable using the thermostatic heating probe. However, even though the SPP became detectable, the states of the feet with ulcer in which SPP could not reach $30 \mathrm{mmHg}$ in both aspects were worse as shown in previous reports on traditional SPP measurement. ${ }^{12,13)}$ On the other hand, the foot in which the SPP became detectable as larger than $30 \mathrm{mmHg}$ did not have ulcer. Additionally, there were no feet that developed ulcer in which the SPP values exceeded $50 \mathrm{mmHg}$ in both aspects of the feet after heating. Thus, the thermostatic heating probe may be useful for distinguishing feet with a poor SPP detection from feet with a truly poor perfusion. An increase in SPP as induced by local heating may serve as a parameter for evaluating the severity of limb ischemia. However, the clinical mean SPP measured using a thermostatic heating probe remained unclear in this study and thus warrants further investigation.

SPP detection is occasionally difficult even in nonsevere ischemic limbs. From the present results, difficulties in SPP measurement may not be completely resolved only with the use of a heating probe. The vasoconstrictor and vasodilator responses of the skin according to temperature are considered stronger in young people than in elderly people. ${ }^{10,14)}$ However, SPP measurement is affected by not only temperature but also many factors involving sympathetic nervous tension. ${ }^{15)}$ The different values in young healthy subjects may have resulted from the strong sympathetic nerve tension offsetting the response by heating. On the other hand, at the plantar aspect, the feet that had shown a high SPP by the plain laser Doppler probe appeared to have a small or no increase in SPP after heating. The plantar aspect of the healthy feet might be sufficiently warm or the SPP might be sufficiently high when the plain probe was used. We could not fully explain this because we did not measure the skin temperature before the measurement using a plain probe in both healthy and ischemic feet. However, for the elderly patients with cold ischemic limbs, a thermostatic heating probe could be useful in preparing the skin temperature condition for measuring SPP in a short warm-up time, as well as for improving the SPP detection rate.

The SPP values measured using the plain laser Doppler probe were statistically similar for both the dorsum and the plantar aspect of the feet in the healthy subjects. However, as skin perfusion and response to temperature are different in each body area, SPP also shows different values by area. ${ }^{16-18)}$ Previous studies have shown that SPP tends to have higher values in the plantar aspect and toe than in the dorsum. ${ }^{16,19)}$ The skin plays a major role in the thermoregulatory function in response to temperature, particularly in hairless skin areas such as the palms, fingertips, plantar aspect of the feet, and toe tips, which have more arteriovenous anastomosis than the other skin areas. $^{20,21)}$ Masaki, et al. previously measured SPP at the dorsum and toe in 20- to 23-year-old subjects. They found that the SPP at the dorsum was $48 \mathrm{mmHg}$, which is not significantly different from the SPP measured in the current study ( $45 \mathrm{mmHg}$ ); the SPP at the toe was 64 $\mathrm{mmHg}$, which is significantly higher than the SPP at the dorsum. ${ }^{19)}$ Castronuovo, et al. measured SPP at the calf, foot, dorsal toe, and plantar toe in normal limbs and then obtained the SPP value at the plantar toe $(73 \mathrm{mmHg})$, which was higher than the SPP value in all other locations. ${ }^{16)}$ On the other hand, Fischer, et al. reported an SPP of $81 \mathrm{mmHg}$ at the dorsum among healthy persons. ${ }^{17}$ Okamoto, et al. reported an SPP of $74 \mathrm{mmHg}$ at the dorsum in dialysis patients without peripheral arterial disease and an SPP of $79 \mathrm{mmHg}$ in healthy individuals. ${ }^{18)}$ These SPP values at the dorsum are higher than the SPP values of the healthy subjects in this study as measured using a thermostatic heating probe. Although the cause of the difference in the SPP values at the dorsum between the previous studies and this study was unclear, age might have affected the results (our healthy subjects showed lower SPP values also at the plantar aspect [45 $\mathrm{mmHg}]$ ); however, a description of the subjects' age in these previous studies was lacking. ${ }^{17,18)}$ In the patients in this study, the detectability of SPP was improved at the plantar aspect but not at the dorsum. 
Such phenomenon at the dorsum was observed in 1 foot suspected to have a truly poor perfusion. However, it is also possible that the difference in response after heating may reflect the distribution of arteriovenous anastomosis.

The results of SPP measurement can be affected by the measurement systems employed. We used PeriFlux System 5000 to measure SPP in this study. Previously, we compared the SPP values measured using Laserdopp Model PV2000 (Vasamedics Inc., USA) and PeriFlux System 5000 in young healthy subjects and PAD patients, and reported that PeriFlux System 5000 obtained a higher SPP value of 4-9 mmHg than Laserdopp Model PV2000. ${ }^{22)}$ As for the difference between both systems, PeriFlux System 5000 reads values by $1 \mathrm{mmHg}$, whereas Laserdopp Model PV2000 reads values by $10 \mathrm{mmHg}$ for values above $50 \mathrm{mmHg}$ and by $5 \mathrm{mmHg}$ for values less than $50 \mathrm{mmHg}$. This means that $79 \mathrm{mmHg}$ as measured using PeriFlux System 5000 may be measured as $70 \mathrm{mmHg}$ when using Laserdopp Model PV2000. Thus, the results may have a maximum difference of $9 \mathrm{mmHg}$ for values larger than $50 \mathrm{mmHg}$, and may have a maximum difference of $4 \mathrm{mmHg}$ for values smaller than $50 \mathrm{mmHg}$. Moreover, the measurement depth of the laser Doppler probe of PeriFlux System 5000 is $0.5-1.0 \mathrm{~mm}$ from the skin surface, which is slightly shallower than that of Laserdopp Model PV2000 (1-2 mm). This factor may affect the measurement.

\section{Conclusion}

In the healthy subjects of this study, the SPP values at the dorsum and plantar aspect of the feet showed no statistically significant difference between the plain laser Doppler probe and the thermostatic heating probe. In the patients with ischemic limb, the SPP values were increased and the detection rates were improved using the thermostatic heating probe. Thus, a thermostatic heating probe may be useful for preparing a temperature condition similar to the skin temperature in order to effectively measure SPP and improve its detection rate in ischemic feet. An increase in SPP as induced by heating may be used as a parameter for evaluating limb ischemia and the skin reserve capacity.

\section{ACKNOWLedgement}

We are indebted to Dr. Edward Barroga for the editorial review of the English manuscript.

\section{Disclosure Statement}

The authors declare that they have no conflicts of interest associated with this study.

\section{REFERENCES}

1) Castronuovo JJ, Adera HM, Smiell JM, et al. Skin perfusion pressure measurement is valuable in the diagnosis of critical limb ischemia. J Vasc Surg 1997; 26: 629-37.

2) Doi T. The structure and the characteristic of the skin and blood vessel of the skin, and the function. Biomedical Thermology 2003; 23: 48-56.

3) Johnson JM, Park MK. Reflex control of skin blood flow by skin temperature: role of core temperature. J Appl Physiol Respir Environ Exerc Physiol 1979; 47: 1188-93.

4) Rendell MS, Giitter M, Bamisedun O, et al. The laser Doppler analysis of posturally induced changes in skin blood flow at elevated temperatures. Clin Physiol 1992; 12: 241-52.

5) Taylor WF, Johnson JM, O'Leary D, et al. Effect of high local temperature on reflex cutaneous vasodilation. J Appl Physiol Respir Environ Exerc Physiol 1984; 57: 191-6.

6) Patel S, Knapp CF, Donofrio JC, et al. Temperature effects on surface pressure-induced changes in rat skin perfusion: implications in pressure ulcer development. J Rehabil Res Dev 1999; 36: 189-201.

7) Mori K, Hayabuchi Y, Kuroda Y, et al. Age-related endothelium-dependent vascular relaxation in rat thoracic aorta in response to colforsin. Pediatr Int 1999; 41: 673-81.

8) Nakahara M, Ichioka S, Shibata M, et al. Effect of local heating on cutaneous microcirculation in mice comparison between age groups. J Jpn Coll Angiol 2004; 44: 109-15.

9) Farrell DM, Bishop VS. The roles of cGMP and cAMP in active thermoregulatory vasodilation. Am J Physiol 1997; 272: R975-81.

10) Kingma BR, Frijns AJ, Saris WH, et al. Cold-induced vasoconstriction at forearm and hand skin sites: the effect of age. Eur J Appl Physiol 2010; 109: 915-21.

11) Matsuo H, Hayashi T, Takeda $Y$, et al. The effects of artificial carbon dioxide foot bathing on the skin of ischemic feet measured by laser Doppler flowmeter. J Jpn Coll Angiol 2000; 40: 923-8.

12) Masaki H, Tabuchi A, Yunoki Y, et al. Collective therapy and therapeutic strategy for critical limb ischemia. Ann Vasc Dis 2013; 6: 27-32.

13) Watanabe Y, Onozuka A, Obitsu Y, et al. Skin perfusion pressure measurement to assess improvement in peripheral circulation after arterial reconstruction for critical limb ischemia. Ann Vasc Dis 2011; 4: 235-40.

14) DeGroot DW, Havenith G, Kenney WL. Responses to 
mild cold stress are predicted by different individual characteristics in young and older subjects. J Appl Physiol 2006; 101: 1607-15.

15) Donadio V, Nolano M, Provitera V, et al. Skin sympathetic adrenergic innervation: an immunofluorescence confocal study. Ann Neurol 2006; 59: 376-81.

16) Castronuovo JJ, Pabst TS, Flanigan DP, et al. Noninvasive determination of skin perfusion pressure using a laser Doppler. J Cardiovasc Surg (Torino) 1987; 28: 253-7.

17) Fischer M, Hoffmann U, Oomen P, et al. Simultaneous measurement of digital artery and skin perfusion pressure by the laser Doppler technique in healthy controls and patients with peripheral arterial occlusive disease. Eur J Vasc Endovasc Surg 1995; 10: 231-6.

18) Okamoto K, Oka M, Maesato K, et al. Peripheral arterial occlusive disease is more prevalent in patients with hemodialysis: comparison with the findings of multidetector-row computed tomography. Am J Kidney Dis 2006; 48: 269-76.

19) Masaki H, Ishida A, Matsumoto $M$, et al. Evaluation of ischemic limb by measuring transcutaneous oxygen tension and skin perfusion pressure. Japanese Society for Limb Salvage Research 2004; 2004: 35-41.

20) Bergersen TK, Hisdal J, Walløe L. Perfusion of the human finger during cold-induced vasodilatation. Am J Physiol 1999; 276: R731-7.

21) Gorgas K, Böck P, Tischendorf F, et al. The fine structure of human digital arterio-venous anastomoses (Hoyer-Grosser's organs). Anat Embryol 1977; 150: 269-89.

22) Kubo H, Masaki H, Tabuchi A, et al. Difference of the skin perfusion pressure (SPP) measuring equipment. J Jpn Coll Angiol. 2011; 51 (Suppl): S128. 